\title{
897.
}

\section{SUR LES RACINES D'UNE ÉQUATION ALGÉBRIQUE.}

[From the Comptes Rendus de l'Académie des Sciences de Paris, t. Cx. (JanvierJuin, 1890), pp. 174-176, 215-218.]

Sort $f(u)$ une fonction rationnelle et entière, avec des coefficients réels ou imaginaires, de l'ordre $n$; en supposant que l'équation $f^{\prime}(u)=0$, de l'ordre $n-1$, ait $n-1$ racines, je démontre que l'équation $f(u)=0$ aura $n$ racines. Pour cela, soit $f(u)=f(x+i y)=P+i Q$ : je suppose que $c$ dénote une quantité positive donnée, et je considère la surface $c-z=P^{2}+Q^{2}$, en attribuant à la coordonnée $z$ des valeurs positives; c'est seulement pour avoir des maxima au lieu de minima, et pour faciliter ainsi l'exposition, que je prends cette surface au lieu de $z=P^{2}+Q^{2}$. On peut donner à $c$ une valeur si grande que la courbe $c=P^{2}+Q^{2}$ soit une courbe fermée qui ne se coupe pas, c'est-à-dire un contour simple: cela étant, on peut se figurer ce contour comme la ligne de rivage d'une île montagneuse; la valeur de $z$ est au plus $=c$, et, en donnant à $z$ une valeur plus petite, $=b$, on a le contour qui correspond à l'altitude $b$ : évidemment, les contours qui correspondent à des altitudes. différentes ne se coupent pas. Il s'agit de prouver que l'ile a précisément $n$ sommets, chacun de l'altitude $c$.

J'écris

$$
\frac{d P}{d x}=X, \quad \frac{d P}{d y}=Y
$$

donc

et, de plus,

$$
\frac{d Q}{d x}=-Y, \quad \frac{d Q}{d y}=X
$$

$$
\frac{d X}{d x}=a, \quad \frac{d X}{d y}=\frac{d Y}{d x}=h, \quad \frac{d Y}{d y}=-a
$$

C. XIII. 
et de là

$$
\begin{aligned}
\frac{d^{2} P}{d x^{2}} & =\quad a, & \frac{d^{2} Q}{d x^{2}} & =-h \\
\frac{d^{2} P}{d x d y} & =\quad h, & \frac{d^{2} Q}{d x d y} & =\quad a \\
\frac{d^{2} P}{d y^{2}} & =-a, & \frac{d^{2} Q}{d y^{2}} & =\quad h
\end{aligned}
$$

Cela étant, nous avons

$$
-\frac{1}{2} \frac{d z}{d x}=P X-Q Y, \quad-\frac{1}{2} \frac{d z}{d y}=P Y+Q X
$$

on aura un plan tangent horizontal pour $P=0, Q=0$, ou pour $X=0, Y=0$; les valeurs $P=0, Q=0$, appartiennent à la valeur $c$ de $z$ et correspondent à des sommets de montagne de cette altitude $c$; les valeurs $X=0, Y=0$ correspondent à des sommețs de col. En effet, nous avons

done

$$
\begin{aligned}
& -\frac{1}{2} \frac{d^{2} z}{d x^{2}}=P a-Q h+X^{2}+Y^{2} \\
& -\frac{1}{2} \frac{d^{2} z}{d x d y}=P h+Q a \\
& -\frac{1}{2} \frac{d^{2} z}{d y^{2}}=-P a+Q h+X^{2}+Y^{2}
\end{aligned}
$$

$$
\begin{aligned}
\frac{1}{4}\left[\frac{d^{2} z}{d x^{2}} \frac{d^{2} z}{d y^{2}}-\left(\frac{d^{2} z}{d x d y}\right)^{2}\right] & =\left(X^{2}+Y^{2}\right)^{2}-(P a-Q h)^{2}-(P h+Q a)^{2} \\
& =\left(X^{2}+Y^{2}\right)^{2}-\left(P^{2}+Q^{2}\right)\left(a^{2}+h^{2}\right)
\end{aligned}
$$

valeur positive pour $P=0, Q=0$; négative pour $X=0, Y=0$.

A présent, nous avons $f^{\prime}(x+i y)=X-i Y$; donc, en supposant que l'équation $f^{\prime}(x+i y)=0$ ait $n-1$ racines, il y aura $n-1$ systèmes de valeurs réelles de $x, y$ qui satisfont aux équations $X=0, \quad Y=0$; pour chaque système, il y aura des valeurs déterminées de $P, Q$ et de là aussi de $z$; ces valeurs de $z$ seront en général différentes. Ainsi il y aura dans l'île $n-1$ cols, dont les altitudes seront en général différentes: soient $c_{1}, c_{2}, \ldots, c_{n-1}$ ces altitudes, commençant avec la plus petite.

Pour $z=0$, nous avons un contour simple, et de même pour une valeur quelconque plus petite que $c_{1}$; mais, pour $z=c_{1}$, nous avons un col; le contour est une courbe, figure de 8 ; en donnant à $z$ une valeur un peu plus grande, le contour se divise en deux courbes fermées, ou bien contours simples, extérieurs l'un à l'autre. Pour $z=c_{2}$, nous avons encore un col; l'un des contours simples s'est changé en figure de 8, et, pour une valeur un peu plus grande, le contour se divise en trois contours simples, chacun extérieur aux autres. En continuant de cette manière, on a, pour $c_{n-1}$, le col le plus haut; le contour est composé de $n-2$ contours simples et d'une figure de 8 ; et, en donnant à $z$ une valeur un peu plus grande, on obtient un contour composé de $n$ contours simples, chacun extérieur aux autres. Enfin, en 
faisant croitre $z$, chacun des contours simples doit se réduire à un point, c'est-à-dire qu'il doit y avoir précisément $n$ sommets de montagne; mais il n'y a pas de sommet de montagne, sinon pour la valeur $z=c$; donc il y a précisément $n$ sommets de montagne, chacun de l'altitude $c$. On suppose toujours que l'équation $f^{\prime}(x+i y)=0$ n'ait pas de racines égales, mais il peut bien arriver que deux ou plusieurs des valeurs $c_{1}, c_{2}, \ldots, c_{n}$ deviennent égales; la démonstration est très peu changée, en donnant à $z$ une valeur un peu plus grande que celle qui correspond à l'altitude des cols d'altitude égale; le contour se divise toujours en contours simples, extérieurs chacun aux autres.

Il va sans dire que cette démonstration repose sur les mêmes principes que celles de Gauss et de Cauchy.

Je reprends la théorie des racines de l'équation $f(u)=0$; au lieu de la surface $c-z=P^{2}+Q^{2}$, il convient de considérer la surface $(c-z)^{2}=P^{2}+Q^{2}$, en faisant attention seulement aux valeurs de $z$ positives et pas plus grandes que $c$. La théorie est. très peu changée; les contours sont les mêmes qu'auparavant, mais ils appartiennent à des altitudes différentes; et, au lieu de maxima $z=c$ pour $P=0, Q=0$, on a des points coniques, c'est-à-dire, dans l'île montagneuse, au lieu d'un sommet arrondi de montagne, on a un cône ou un pic.

Mais avec la nouvelle surface, on construit graphiquement l'approximation de Newton: partant d'une valeur réelle ou imaginaire approximative $u$, on obtient la nouvelle valeur

$$
u_{1}=u+h=u-\frac{f(u)}{f^{\prime}(u)}
$$

Je représente $u$ par le point $(x, y, z)$ de la surface $(c-z)^{2}=P^{2}+Q^{2}$, ou le point $(x, y)$ du plan des sommets $z-c$; et, de même, $u_{1}$ par le point $\left(x_{1}, y_{1}, z_{1}\right)$ de la surface, ou $\left(x_{1}, y_{1}\right)$ du plan des sommets: cela étant, si, par le point $(x, y, z)$ de la surface, on mène la droite de plus grande pente (droite tangente à la surface et perpendiculaire au contour), cette droite rencontrera le plan des sommets en un point $\left(x_{1}, y_{1}\right)$, et l'on obtient ainsi le point $\left(x_{1}, y_{1}, z_{1}\right)$ de la surface, qui représente la valeur cherchée $u_{1}$. En particulier, si les coefficients de $f(u)$ sont réels; on a

l'équation $(c-z)^{2}=P^{2}+Q^{2}$ devient

$$
Q=0
$$

c'est-à-dire

ou enfin

$$
(c-z)^{2}=P^{2},
$$

$$
c-z= \pm P
$$

$$
c-z= \pm f(x)
$$

et la section verticale de l'île est formée par des parties de ces deux courbes symétriques: pour la théorie géométrique, on peut évidemment y substituer la seule courbe $c-z=f(x)$. 
J'ai proposé, il y a plus de dix ans (Amer. Math. Journ., t. II., 1879, [694]), le problème que je nomme "The Newton-Fourier imaginary Problem," et dans une Note (Quart. Math. Journ., t. xvI., 1879, [736]), "Application of the Newton-Fourier method to an imaginary root of an equation," j'ai considéré le cas d'une équation quadratique. Pour l'équation $u^{2}-1=0$, on a

cela donne

$$
u_{1}=u-\frac{u^{2}-1}{2 u}=\frac{1}{2}\left(u+\frac{1}{u}\right)
$$

et de là

$$
u_{1}-1=\frac{1}{2 u}(u-1)^{2}, \quad u_{1}+1=\frac{1}{2 u}(u+1)^{2},
$$

$$
\frac{u_{1}-1}{u_{1}+1}=\left(\frac{u-1}{u+1}\right)^{2}
$$

Cette dernière équation a rapport aux deux racines +1 et -1 , et, quoiqu'elle donne les résultats les plus élégants, cependant, en vue de la théorie générale, il vaut mieux considérer l'équation $u_{1}-1=\frac{1}{2 u}(u-1)^{2}$ qui se rapporte à la seule racine +1 .

Je remarque d'abord que la formule originale $u_{1}=\frac{1}{2}\left(u+\frac{1}{u}\right)$ donne

$$
x_{1}=\frac{1}{2} x\left(1+\frac{1}{x^{2}+y^{2}}\right), \quad y_{1}=\frac{1}{2} y\left(1-\frac{1}{x^{2}+y^{2}}\right)
$$

donc les valeurs de $x$ et $x_{1}$ seront à la fois positives ou négatives, et ainsi l'on peut ne faire attention qu'aux valeurs positives. Cela étant, nous avons

$$
x_{1}+i y_{1}-1=\frac{(x+i y-1)^{2}}{2(x+i y)} \text {. }
$$

Désignons par $A$ le point $(0,1)$, par $B$ le point $(0,-1)$, par 0 le point $(0,0)$; et aussi par $P$ le point $(x, y)$, et de même par $P_{1}$ le point $\left(x_{1}, y_{1}\right)$; écrivons aussi $x+i y=s e^{i \phi}, x+i y-1=r e^{i \theta}, x_{1}+i y_{1}=r_{1} e^{i \theta}$; l'équation est

done

$$
r_{1} e^{i \theta_{1}}=\frac{1}{2} \frac{r^{2} e^{2 i \theta}}{s e^{2 \phi}}
$$

$$
r_{1}=\frac{1}{2} \frac{r^{2}}{s}, \quad\left(c^{\prime} \text { est-à-dire } A P_{1}=\frac{A P^{2}}{2 O P}\right)
$$

et

$$
\theta_{1}=2 \theta-\phi, \quad\left(\text { c'est-à-dire } \widehat{A P_{1} x}=\widehat{2 A P x}-\widehat{O P x}\right) \text {. }
$$

Je remarque que, dans la géométrie des vecteurs, la seule équation $A P_{1}=\frac{A P^{2}}{20 P}$ dénote l'équation en $x_{1}+i y_{1}, x+i y$, c'est-à-dire les deux équations que je viens de trouver.

Partant d'un point quelconque $P$, on obtient une suite de points $P_{1}, P_{2}, P_{3}, \ldots$; et, si le point $P$ est sur l'axe des $y(x=0)$, tous les autres points seront aussi sur l'axe de $y$, et l'on n'approche ni du point $A$ ni du point $B$. Mais, si la coordonnée $x$ a une valeur positive si petite que l'on veut, on arrive enfin infiniment près du 
point $A$, et l'on peut même (dans un sens qui sera expliqué plus bas, mais qui n'est pas le sens le plus naturel) dire que l'approximation est régulière. En effet, on n'a pas toujours $A P_{1}<A P$, et ainsi, dans le sens le plus naturel, l'approximation n'est pas toujours régulière. Pour étudier cela, j'écris $A P_{1}=A P$; cela donne $A P=20 P$, ou, ce qui est la même chose, $x^{2}+y^{2}+\frac{2}{3} x=\frac{1}{3}$, c'est-à-dire que le point $P$ sera situé sur le cercle, centre $x=-\frac{1}{3}$ et rayon $=\frac{2}{3}$; en ne faisant attention qu'aux valeurs positives de $x$, on a un segment compris entre l'axe des $y$ et un arc par les points $\left(x=0, y= \pm \frac{1}{\sqrt{ } 3}\right),\left(x=\frac{1}{3}, y=0\right)$. Si le point $P$ est sur l'arc, on aura $A P_{1}=A P$; si $P$ est en dedans du segment, alors $A P_{1}>A P$; si $P$ est en dehors du segment, $A P_{1}<A P$.

Mais, en supposant $P$ en dehors du segment, et ainsi $A P_{1}<A P$, il peut bien arriver que $P_{1}$ soit en dedans du segment, et, cela étant, on aura $A P_{2}>A P_{1}$, et l'approximation ne sera pas régulière. Mais, en considérant le cercle $x^{2}+y^{2}-\frac{2}{3} x=\frac{1}{3}$, lequel est le cercle, centre $A$ et rayon $\frac{2}{3}$, qui touche le segment au point $\left(x=\frac{1}{3}, y=0\right)$, alors, en supposant que le point $P$ soit en dedans de ce cercle, on aura $A P_{1}<A P$, le point $P_{1}$ sera aussi en dedans du cercle, et ainsi en dehors du segment; et les points successifs $P, P_{1}, P_{2}, \ldots$ approcheront continuellement du point $A$; l'approximation sera dans ce cas régulière.

Il y a ainsi trois régions: le segment, le cercle $x^{2}+y^{2}-\frac{2}{3} x=\frac{1}{3}$ et le résidu du demi-plan; on pourrait les nommer régions noire, blanche et grise respectivement. C'est seulement pour un point $P$ à l'intérieur de la région blanche que l'approximation est certainement régulière.

Nous venons de considérer en effet les cercles qui ont pour centre le point $A ; A P_{1}<A P$ veut dire que le point $P$ est situé sur un cercle plus grand, et $P_{1}$ - sur un cercle plus petit; mais, au lieu de ces cercles concentriques, considérons des cercles quelconques qui entourent le point $A$, sans se couper les uns les autres; et convenons de dire que c'est un bon pas quand on passe du point $P$ sur un cercle plus grand à un point $P_{1}$ sur un cercle plus petit: avec cette convention on aura, en général, trois régions, lesquelles cependant ne seront pas les mêmes comme auparavant. En particulier, si nous considérons les cercles $A P=k B P$ ( $k$ une constante quelconque plus petite que l'unité), alors il n'y a pas de région noire, ou, si l'on veut, la région noire se réduit à la seule droite $y=0$; donc il n'y a pas non plus de région grise, et le demi-plan entier est région blanche, c'est-à-dire, dans le sens que je viens d'expliquer, l'approximation est toujours régulière. En effet, c'eșt là la théorie à laquelle on est conduit au moyen de l'équation $\frac{u_{1}-1}{u_{1}+1}=\left(\frac{u-1}{u+1}\right)^{2}$ ci-dessus mentionnée.

En parlant de cercles, j'ai fait une restriction qui n'est nullement nécessaire; j'aurais pu parler d'ovales, de forme quelconque, qui entourent le point $A$ sans se couper les uns les autres.

J'espère appliquer cette théorie au cas d'une équation cubique, mais les calculs sont beaucoup plus difficiles. 